\title{
Phosphatidylinositol-4-phosphate 5-kinase activity is stimulated during temperature- induced morphogenesis in Candida albicans
}

\author{
Michelle L. Hairfield, ${ }^{1}$ Caroline Westwater ${ }^{2}$ and Joseph W. Dolan ${ }^{1,2}$
}

Author for correspondence: Joseph W. Dolan. Tel: +1 843792 3104. Fax: +1 8437922464.

e-mail:dolanjw@musc.edu

Molecular and Cellular Biology Program¹, Division of Mycology22, Department of Microbiology and Immunology, Medical University of South Carolina, 173 Ashley Avenue, PO Box 250504, Charleston, SC 29403, USA

\begin{abstract}
Phosphoinositides are important lipid signalling molecules in eukaryotic cells. Phosphatidylinositol-4-phosphate 5-kinase (PI4P5K) catalyses the production of phosphatidylinositol 4,5-bisphosphate $\left(\mathrm{PIP}_{2}\right)$, which stimulates phospholipase D1 (PLD1) activity in mammalian and yeast cells. PLD1 catalyses the formation of phosphatidic acid (PA), which has been shown to activate PI4P5Ks in mammalian and Saccharomyces cerevisiae cells. In the present study, PI4P5K activity in the opportunistic pathogen Candida albicans was identified. A gene with significant sequence homology to the S. cerevisiae PI4P5K was cloned and designated MSS4. This gene was demonstrated to encode a functional PI4P5K by expression in $S$. cerevisiae. This enzyme was found to be membraneassociated and was stimulated by PA. Within the first $\mathbf{2 0}$ min after induction of polarized hyphal growth induced by a shift to elevated temperature, PI4P5K activity increased 2.5-fold. This stimulation was not observed when hyphae were induced by a combination of elevated temperature and serum. A lack of PLD1 activity resulted in the loss of induction of PI4P5K activity during the morphogenetic switch. Furthermore, the addition of propranolol attenuated the stimulation of PI4P5K activity during morphogenesis. These results suggest that PA derived from PLD1 activity stimulates C. albicans PI4P5K during the switch to the hyphal form under some conditions.
\end{abstract}

Keywords: dimorphic transition, phospholipase D, PLD1, MSS4

\section{INTRODUCTION}

Phosphoinositides are found in all eukaryotic cells and are crucial molecules in many cellular processes (Majerus, 1992). These molecules represent only a small portion of the total membrane lipid content. Nevertheless they have emerged as key players in cytoskeletal organization, cell proliferation, vesicular trafficking and lipid signal transduction pathways (reviewed by Fruman et al., 1998; Odorizzi et al., 2000). A vital component in phosphoinositide signalling is phosphatidylinositol 4,5bisphosphate $\left(\mathrm{PIP}_{2}\right)$. PIP 2 has a fundamental role in many cellular processes. One of the most significant roles for $\mathrm{PIP}_{2}$ is as a source for the important second messengers 1,2-diacylglycerol (DAG) and inositol 1,4,5trisphosphate following hydrolysis by phospholipase $\mathrm{C}$

Abbreviations: DAG, 1,2-diacylglycerol; LPP, lipid phosphate phosphohydrolases; PA, phosphatidic acid; PI4P5K, phosphatidylinositol-4-phosphate 5-kinase; $\mathrm{PIP}_{2}$, phosphatidylinositol 4,5-bisphosphate; PLC, phospholipase C; PLD1, phospholipase D1.
(PLC). DAG is known to activate several isoforms of protein kinase $\mathrm{C}$ in mammalian systems and is important in cellular secretion (Liscovitch et al., 1999; Siddhanta \& Shields, 1998), while inositol 1,4,5-trisphosphate releases intracellular calcium stores. In addition, $\mathrm{PIP}_{2}$ regulates the actin cytoskeleton by binding and regulating various actin-binding proteins (Janmey, 1994) such as profilin (DiNubile \& Huang, 1997a, b), gelsolin (Janmey \& Stossel, 1987) and $\alpha$-actinin (Fukami et al., $1992)$ in vertebrates. The modulation of the activity of other proteins is another function of $\mathrm{PIP}_{2}$ : phospholipase D1 (PLD1) activity is stimulated by $\mathrm{PIP}_{2}$ in mammalian (Liscovitch et al., 1994), Saccharomyces cerevisiae (Rose et al., 1995; Waksman et al., 1996) and Candida albicans cells (McLain \& Dolan, 1997). Roles for proteins that contain a pleckstrin homology domain have also been described in $\mathrm{PIP}_{2}$ signalling. It has been demonstrated, for example, that the PLC $\delta 1$ pleckstrin homology domain has a high affinity for PIP ${ }_{2}$ (Isakoff et al., 1998) and that the pleckstrin homology domain is necessary and sufficient for PLC $\delta 1$ attachment to membranes 
(Varnai \& Balla, 1998). Due to the wide range of roles played by $\mathrm{PIP}_{2}$, its synthesis and degradation must be tightly regulated by the cell.

$\mathrm{PIP}_{2}$ is synthesized by the sequential phosphorylation of phosphatidylinositol by phosphatidylinositol-4-kinase and phosphatidylinositol-4-phosphate 5-kinase (PI4P5K) at the D-4 and D-5 position of the inositol ring, respectively. PIP ${ }_{2}$ can also be synthesized by phosphatidylinositol-5-phosphate 4-kinase (from phosphatidylinositol 5-phosphate) (Rameh et al., 1997). Originally, type I and type II PI4P5Ks were described in mammals (Loijens \& Anderson, 1996); however, the substrate specificity of these enzymes has revealed that the type II enzymes are phosphatidylinositol-5-phosphate 4-kinases (Rameh et al., 1997). In mammals, different isoforms of PI4P5K have been identified and designated $\alpha, \beta$ and $\gamma$ (Ishihara et al., 1996, 1998; Loijens \& Anderson, 1996; Loijens et al., 1996). Acidic phospholipids such as phosphatidic acid (PA) (Ishihara et al., 1996, 1998; Jenkins et al., 1994; Moritz et al., 1992) and phosphatidylserine (Cochet \& Chambaz, 1986) have been shown to stimulate PI4P5K in mammalian cells. Additional regulators of PIP $_{2}$ synthesis include the small GTP-binding proteins Rho and Rac (Chong et al., 1994; Tolias et al., 2000). Honda et al. (1999) demonstrated recently that PI4P5K $\alpha$ is a downstream effector of ADP-ribosylation factor ARF6 and is involved in membrane ruffling.

The S. cerevisiae PI4P5K is encoded by the gene MSS4 and is a 779 aa protein (Desrivieres et al., 1998; Homma et al., 1998). The yeast PI4P5K is required for actin cytoskeleton assembly as mutants fail to form normal actin filaments and fail to localize the cytoskeleton properly during polarized cell growth (Desrivieres et al., 1998; Dove et al., 1997; Homma et al., 1998). Loss of PI4P5K is suppressed by overexpression of $\mathrm{RHO} 2$, a Rho-type GTPase involved in the regulation of the actin cytoskeleton, implicating Rho2p as a downstream effector of PI4P5K (Desrivieres et al., 1998). As in mammalian systems, PI4P5K in S. cerevisiae has been reported to be stimulated by PA (Homma et al., 1998). PA is a putative second messenger that has been implicated in the regulation of lipid metabolism (Liscovitch et al., 1999), cell proliferation (Kaszkin et al., 1996), differentiation (Amsterdam et al., 1994), secretion (Siddhanta \& Shields, 1998) and actin polymerization (Ha \& Exton, 1993). PA can be produced by the hydrolysis of the major membrane phospholipid, phosphatidylcholine, by signal-activated PLD1. Since PI4P5K catalyses the production of $\mathrm{PIP}_{2}$, which is required for the maximal activity of PLD1, and PLD1 catalyses the production of $\mathrm{PA}$, which is required for the maximal activity of PI4P5K, the sequential action of PLD1 and PI4P5K could result in a feedback loop that would stimulate the production of both PA and PIP $_{2}$ (Liscovitch et al., 1994).

C. albicans is the most frequently isolated fungal pathogen in humans. Infections caused by this organism are a major cause of morbidity and mortality among immunocompromised patients, women of childbearing age, infants and the elderly. Antifungal antibiotics are available; however, many have the potential to cause liver and/or kidney toxicity in humans. Strains of C. albicans that are resistant to the most commonly used antifungals are appearing with increasing frequency (Rex et al., 1995). It is therefore essential that the mechanisms involved in C. albicans virulence be elucidated. This organism can grow as budding yeast-form cells or as elongated hyphae, depending on growth conditions. Virulence has been associated with the ability to undergo conversion from the round yeastform to the elongated hyphal form in a morphogenetic process termed dimorphic transition (Cutler, 1991; Navarro-Garcia et al., 2001). High temperature, neutral $\mathrm{pH}$ and serum are potent inducers of dimorphic transition (Ernst, 2000; Odds, 1988). The initial stage of dimorphic transition is the formation of a germ tube, which is an elongated daughter cell formed by an existing yeast-form cell. It has been proposed that hyphae have a mechanical advantage in the penetration of host-cell membranes and the destruction of phagocytes (Odds, 1988). Consequently, molecules found to regulate the process of dimorphic transition may be effective targets for improved antifungal drugs (Leberer et al., 1997). In this report, PI4P5K activity in C. albicans is described. This enzyme is stimulated by PA and during temperature-induced morphogenesis. Finally, we present evidence that stimulation during morphogenesis requires PLD1 activity.

\section{METHODS}

Strains, media and preparation of extracts. C. albicans strains used were CAF2-1 (ura3:: imm434/URA3) and JDC12 (strain also designated pld1 1 in Hube et al., 2001; ura3::imm434/ ura3::imm 434 pld1::hisG::URA3::hisG/pld1::hisG), which are isogenic except as indicated (Hube et al., 2001). S. cerevisiae strains used were $23567(a / \alpha$ his $3 \Delta 1 /$ his $3 \Delta 1$ leu $2 \Delta 0 /$ leu $2 \Delta 1$ met $15 \Delta 0 /+$ lys $2 \Delta 0 /+$ ura $3 \Delta 0 /$ ura $3 \Delta 0$ mss $4:: \mathrm{Kan}^{\mathrm{R}} /$ + ; Research Genetics), SEY6210.1 (MATa leu2-3,112 ura3-52 his3- 200 trp1- 4901 lys2-801 suc2- $\Delta$ ) and AAY202.1 (SEY6210.1 with mss44::HIS3MX6 carrying YEplac-mss4102). Stocks were maintained on YPD agar (Sherman, 1991; Difco), grown in YPD broth at $30{ }^{\circ} \mathrm{C} ; 26{ }^{\circ} \mathrm{C}$ was used for the mss 4-102 temperature-sensitive mutants. $S$. cerevisiae strains were transformed with plasmid pCW20 and empty vector (YEp352) using a lithium acetate protocol (Gietz \& Woods, 2001). Whole-cell extracts were prepared as described previously (McLain \& Dolan, 1997). For localization experiments, CAF2-1 whole-cell extract was fractionated by centrifugation at $10000 \mathrm{~g}$ for $30 \mathrm{~min}$. The resulting soluble and pellet fractions were assayed for PI4P5K activity. For determination of detergent solubility, Triton X-100 was added to $1 \%(\mathrm{w} / \mathrm{v})$ to the whole-cell extract prior to centrifugation.

Morphogenesis assay. To measure the effect of the yeast-tohyphae transition on enzyme activity, PI4P5K and PLD1 in vitro assays were performed with extracts from wild-type cells induced to form hyphae by temperature shift from 30 to $37^{\circ} \mathrm{C}$. Overnight cultures of CAF2-1 and JDC12 in YPD at $30^{\circ} \mathrm{C}$ were diluted 1:100 in fresh YPD medium and incubated at $37^{\circ} \mathrm{C}$ for $90 \mathrm{~min}$. Samples were taken at increasing times post-induction and whole-cell extracts were prepared and assayed for both PI4P5K and PLD1 activities. Where indicated, morphogenesis was induced by temperature shift and the addition of fetal bovine serum to a final concentration of $5 \%$ (v/v). 
PI4P5K assay. PI4P5K activity was assayed according to published methods used for the $S$. cerevisiae enzyme (Homma et al., 1998). Briefly, C. albicans whole-cell extracts containing $55 \mu \mathrm{g}$ protein, as determined by the Bradford assay, were incubated with $80 \mu \mathrm{M}$ PI4P (Sigma) and $5 \mu \mathrm{Ci}\left[\gamma^{32} \mathrm{P}\right] \mathrm{ATP}$ (NEN) for $10 \mathrm{~min}$ at $30^{\circ} \mathrm{C}$. Reactions were incubated with or without $50 \mu \mathrm{M}$ PA where explicitly stated. The reactions were terminated and the lipids extracted by the addition of chloroform/methanol according to the method of Bligh \& Dyer (1959). The lipid products were separated on a silica gel thin layer chromatography plate impregnated with $1.2 \%$ potassium oxalate $(\mathrm{v} / \mathrm{v})$ and developed with chloroform/ methanol/sodium hydroxide/water $(40: 42: 5: 10$, by vol.) (Homma et al., 1998). Quantification of radioactivity incorporated into products was performed with a Molecular Dynamics PhosphorImager. A second assay for PI4P5K used the substrate NBD-PI4P (Echelon Laboratories), which is a fluorescent analogue of the in vivo substrate for PI4P5K. Micelles containing the substrate were prepared as described for the fluorescent PLD1 substrate by McLain \& Dolan (1997) and the reactions were performed as with the radioactive assay. Control samples were always run on the same TLC plate as the experimental samples and both control and experimental samples were scanned at the same time. Values were normalized to controls on the same TLC plate and these normalized values were used to compare results between plates. Statistical analysis was performed using the Student's $t$ test for three independent experiments with duplicate samples in each.

Fluorescent PLD1 assay. PLD1 activity was assayed using whole-cell extracts and the fluorescent substrate BODIPYphosphatidylcholine as described previously (McLain \& Dolan, 1997).

DNA sequence analysis. Homology searches were conducted with the BLAST algorithm. Sequence alignments were performed with DNASTAR MEGALIGN software version 1.05 (DNASTAR) using the CLUSTAL w 1.6 method. A rooted phylogenetic tree was generated using the CLUSTAL $\mathrm{W}$ method with the PAM250 residue weight table.

Construction of plasmids. The DNA encoding the putative $C$. albicans Mss4p was amplified from C. albicans SC5314 genomic DNA by PCR. The primers amplifying the upstream untranslated and $5^{\prime}$ coding region of MSS4 included restriction sites EcoRI and XbaI at the $5^{\prime}$ ends for cloning. Primer sequences were 5'-GCATATGAATTCCATCGCTCTCCATCAACACC-3' and 5'-GAACATCTAGAAACTGCCACTC-3' which contained the restriction sites EcoRI and XbaI (bold type), respectively. The digested PCR product was cloned into the EcoRI-XbaI sites of the S. cerevisiae/E. coli shuttle vector YEp352, generating pCW10. The 3' coding region of MSS4 and its terminator were amplified using primers 5'-GCAGTTTCTAGATGTTCTGGAG-3' and 5'CATGGCAAGCTTTCGGTTGGTCTGAATCAC-3' which contained the restriction sites XbaI and HindIII (bold type), respectively. The digested PCR product was inserted into the $\mathrm{XbaI} /$ HindIII sites of pCW10 to generate plasmid pCW20.

\section{RESULTS}

\section{C. albicans PI4P5K is regulated by PA}

PI4P5K activity is readily detected in S. cerevisiae using a radioactive kinase assay described by Homma et al. (1998). To determine the level of PI4P5K activity in C. albicans, cells were assayed for this kinase as described previously (Homma et al., 1998). PI4P5K activity is
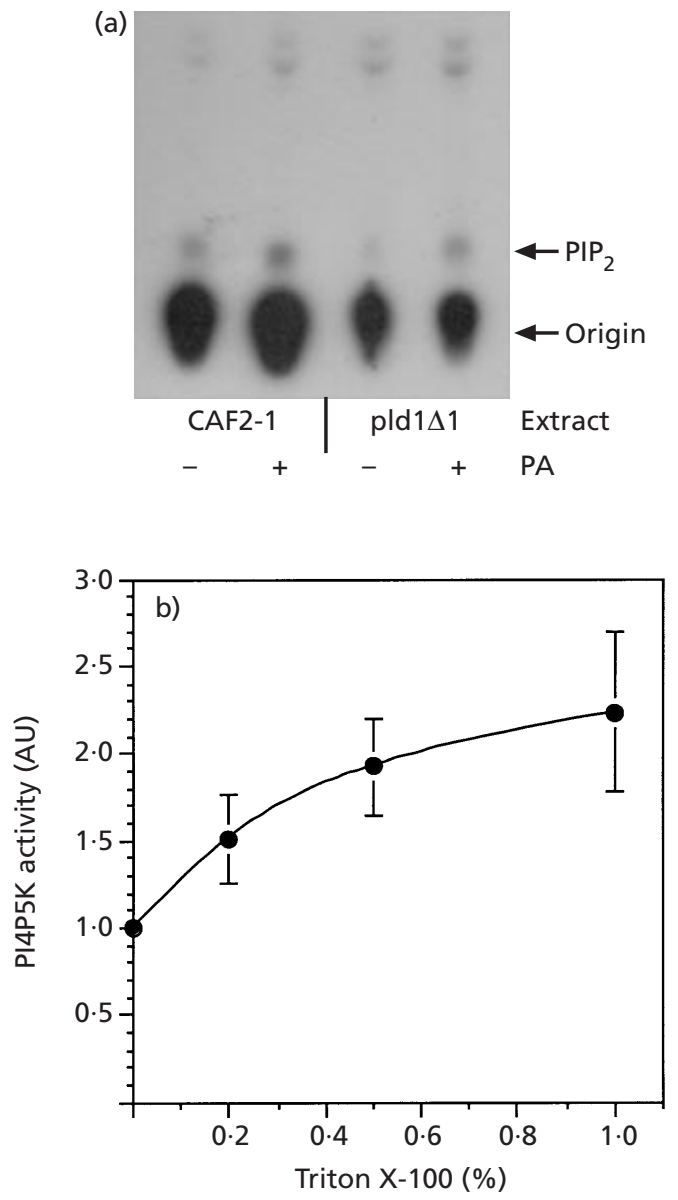

Fig. 1. C. albicans PI4P5K is stimulated by PA and by Triton $X-100$. (a) Autoradiograph of a representative TLC plate showing the effects of PA on PI4P5K activity. (b) The effect of Triton $\mathrm{X}-100$ on PI4P5K activity. PI4P5K activity is presented in arbitrary units (AU) based on the intensity of fluorescence of the NBD$\mathrm{PIP}_{2}$ spots.

stimulated by PA in other organisms (Desrivieres et al., 1998; Homma et al., 1998; Ishihara et al., 1996, 1998; Jenkins et al., 1994; Moritz et al., 1992); therefore, PA was added to reactions to ascertain whether the PI4P5K in C. albicans was stimulated by acidic phospholipids. The results indicated a $48 \%$ increase in the formation of radioactive $\mathrm{PIP}_{2}$ in extracts incubated with exogenous PA as compared to control extracts $(n=6$; $P=0.036$ ) (Fig. 1a). Thus, as with the mammalian and $S$. cerevisiae enzymes, C. albicans expresses a PI4P5K that requires PA for maximal activity.

\section{PI4P5K is membrane-associated}

To examine the intracellular localization of PI4P5K, we first analysed the effect of Triton X-100 on PI4P5K activity (Fig. 1b). Similar to published results for the Schizosaccharomyces pombe enzyme (Vancurova et al., 1999), our results show a stimulation by Triton X-100 at concentrations above $1 \%(\mathrm{v} / \mathrm{v})$. Cell fractionation experiments were subsequently performed. In the absence of detergent, the pellet (particulate) fraction 

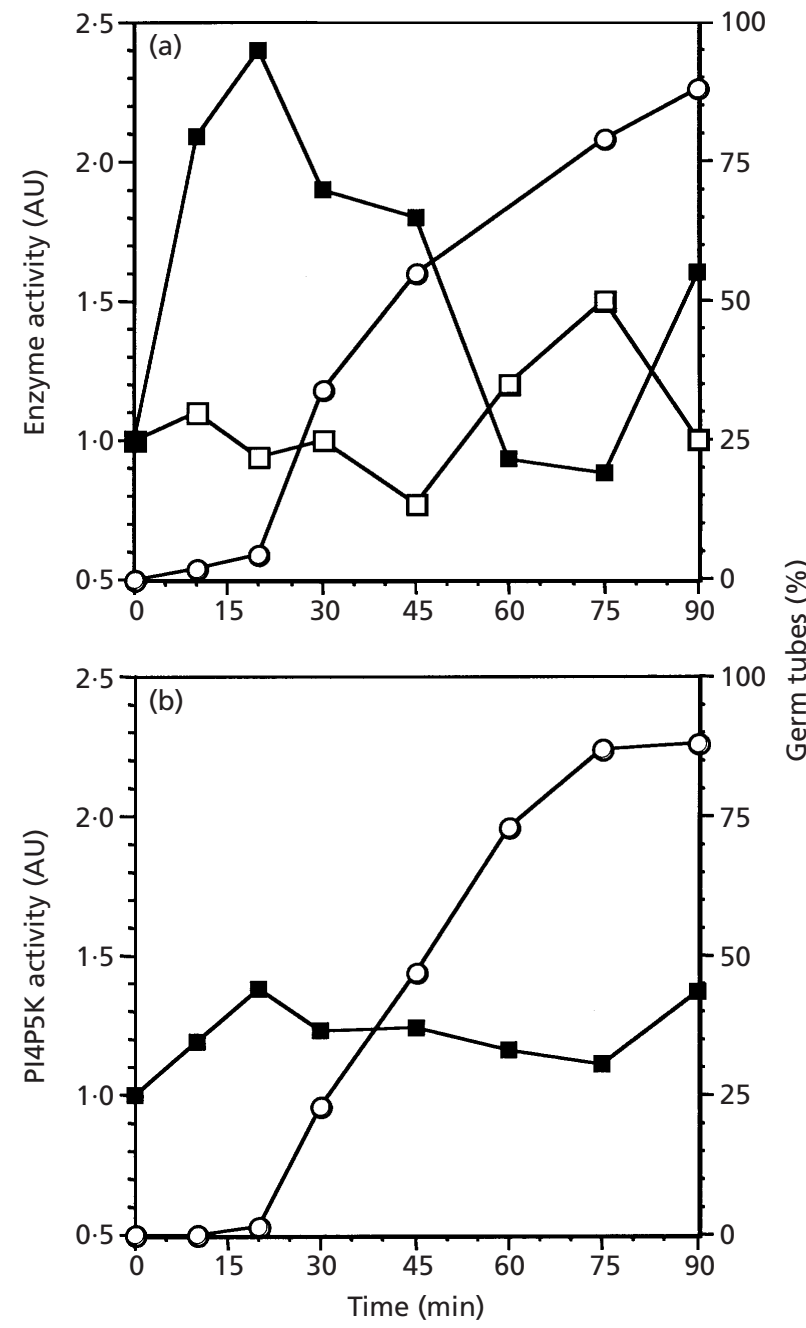

Fig. 2. Both $P I 4 P 5 K$ and PLD1 activity increase in cultures undergoing morphogenesis. (a) Wild-type C. albicans cultures were induced to undergo the switch to polarized hyphal growth. Data shown are from a single representative experiment because the absolute timing of the stimulation varied slightly between experiments. The overall pattern and magnitude of the changes in activity were highly reproducible $(n=6)$. PI4P5K activity $(\boldsymbol{\square})$ is presented in arbitrary units (AU) based on the intensity of fluorescence of the NBD-PIP ${ }_{2}$ spots. PLD1 activity $(\square)$ is stimulated during morphogenesis. Wholecell extracts of cultures induced to form germ tubes by a temperature shift from 30 to $37^{\circ} \mathrm{C}$ were assayed for PLD1 activity at various time points post-induction. PLD1 activity is presented in arbitrary units (AU) based on the intensity of fluorescence of the BODIPY-PA spots. The percentage of cells with germ tubes is indicated at each time point (O). (b) PI4P5K activity was measured in cells that had been induced by both temperature and serum. Samples were taken at time points after induction and the formation of germ tubes $(O)$ and PI4P5K activity ( $\boldsymbol{\square})$ were determined. Data are from a representative experiment.

contained $67.7 \%$ of the total PI4P5K activity, whereas the soluble fraction contained $32.3 \%$ of the total activity. When Triton X-100 was added to solubilize membrane proteins prior to fractionation, PI4P5K activity in the particulate fraction decreased to $27 \%$,
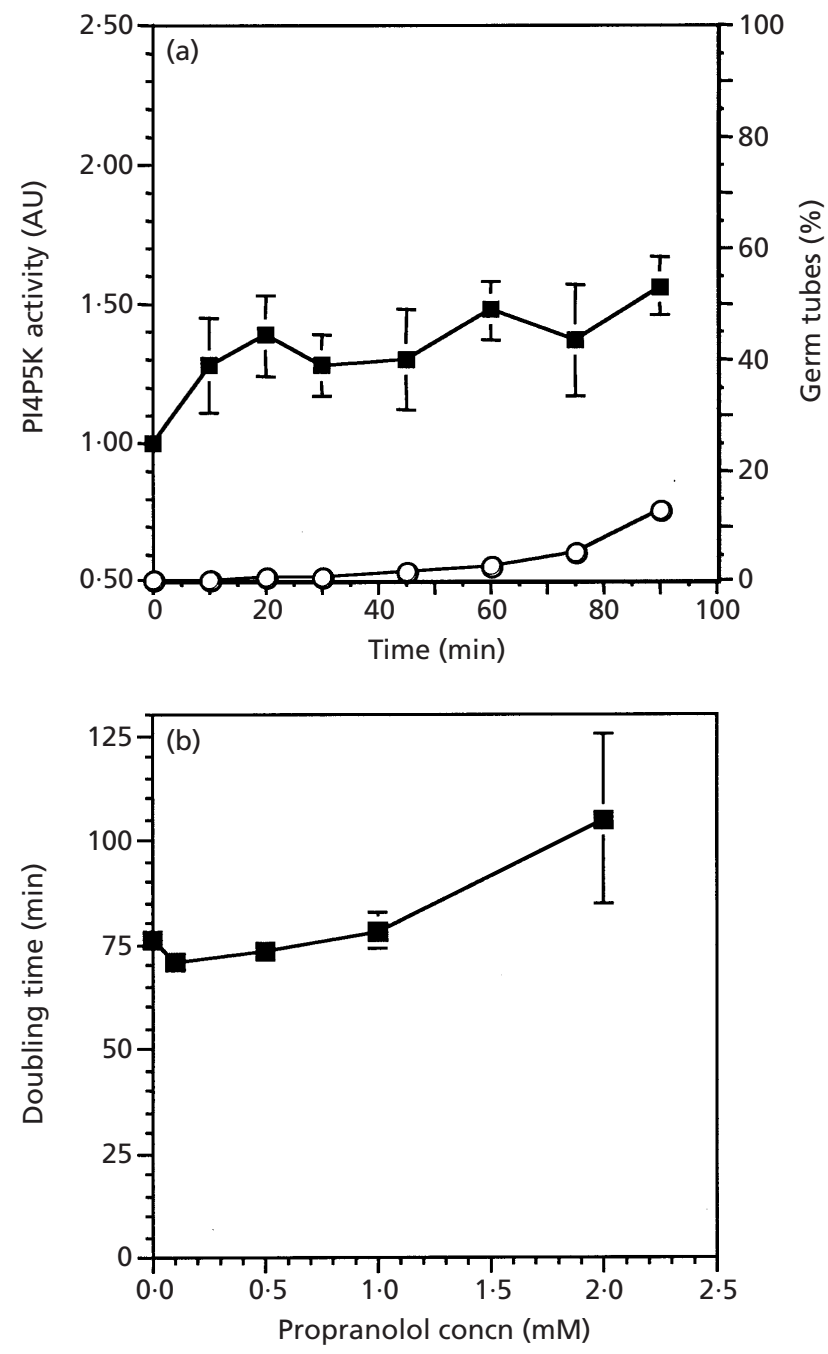

Fig. 3. Propranolol inhibits PI4P5K activation during dimorphic transition. Wild-type C. albicans cultures were induced to form germ tubes by a temperature shift from 30 to $37^{\circ} \mathrm{C}$. Propranolol $(2 \mathrm{mM})$ was added to cultures at the temperature shift. (a) PI4P5K activity ( $\boldsymbol{\square}$ ) was attenuated with the addition of propranolol in comparison with control cells from Fig. 2. Results presented are from a single representative experiment. ( $O$ ), Germ-tube formation. (b) The doubling time was established to verify that propranolol addition did not inhibit germ-tube formation by increasing the generation time. A slight increase in growth rate was noted with $2 \mathrm{mM}$ propranolol which was statistically insignificant. Data are from three independent sets of extracts with each extract assayed twice (total of six assays for each condition). Error bars indicate the standard error of the mean.

which coincided with an increase in activity in the Triton X-100-soluble fraction to $73 \%$ of the total activity. The presence of PI4P5K in the Triton X-100solubilized fraction indicates that the enzyme is associated with membranes. Although the specific membrane to which PI4P5K is bound has not been identified, these data are consistent with the plasma membrane localization reported for the S. cerevisiae (Homma et al., 1998), Schizosaccharomyces pombe (Vancurova et al., 1999) and mammalian enzymes (Honda et al., 1999). 


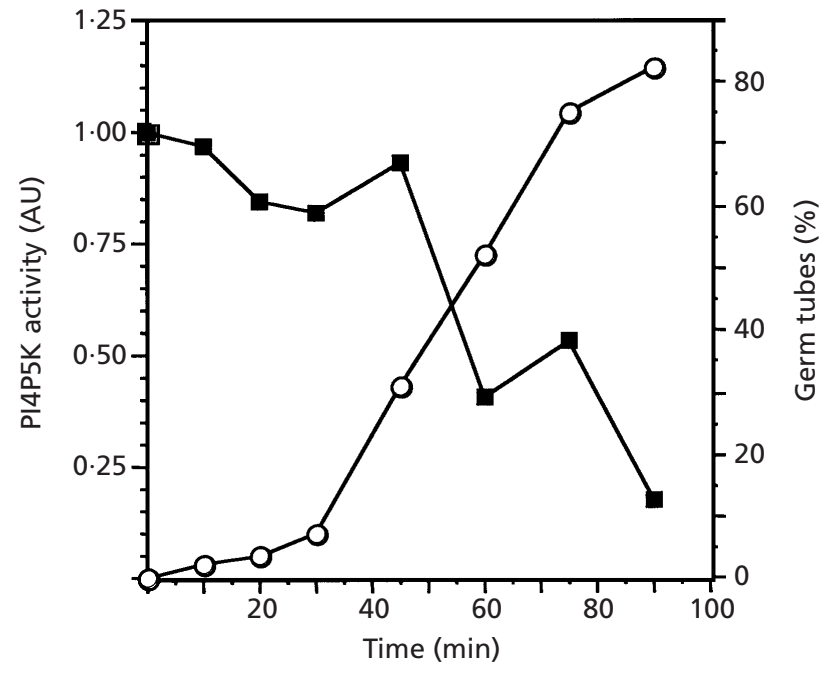

Fig. 4. $P I 4 P 5 K$ is not induced in PLD1-deficient cell cultures undergoing morphogenesis. pld1 $\Delta$ mutant cultures were induced to undergo dimorphic transition by temperature shift.

, PI4P5K activity; $\bigcirc$, percentage of cells with germ tubes. Data shown are from a single representative experiment because the absolute timing of the stimulation varied slightly between experiments. The overall pattern and magnitude of the changes in activity were reproducible. PI4P5K activity is presented in arbitrary units based on the intensity of fluorescence of the NBD-PIP ${ }_{2}$ spots.

\section{PI4P5K activity is stimulated during morphogenesis}

Earlier experiments performed in this laboratory have shown that PLD1 plays a significant role in the morphogenetic process (Hube et al., 2001; McLain \& Dolan, 1997). Given that the product of PLD1 catalysis is PA and that PA stimulates PI4P5K, we aimed to assess a possible correlation between PI4P5K and PLD1 during the morphogenetic switch. C. albicans whole-cell extracts were assayed during morphogenesis to determine the level of PI4P5K activity at various time points following induction. These experiments were performed with a fluorescent assay for PI4P5K activity using NBDPI4P as the substrate for the reaction. The results indicated a maximum level of PI4P5K activity at the 20 min time point, yielding a $2 \cdot 5$-fold increase in activity (Fig. 2a); this increase over the zero time point was statistically significant $(P<0 \cdot 01 ; n=6)$. Maximum PI4P5K activity was coincident with the initiation of germ tube emergence. At $90 \mathrm{~min}$ post-induction there was another increase in PI4P5K activity when $\sim 90 \%$ of cells exhibited germ tubes. In a parallel experiment, cells were inoculated into fresh medium and incubated at $30{ }^{\circ} \mathrm{C}$ to detect changes in PI4P5K due to a return to growth. No significant changes were detected in PI4P5K activity under these conditions (data not shown). The small magnitude of stimulation raises the question of physiological impact. By way of comparison, PLD1 activity is stimulated only $60 \%$ during morphogenesis, but the null mutant is unable to form hyphae under specific conditions and is avirulent in a mouse model system (Hube et al., 2001; McLain \& Dolan, 1997). Thus, there is a solid precedent, supported by both biochemical and genetic analysis, for small changes in activity having relevant and significant physiological impact in the process of morphogenesis in C. albicans.

To determine whether the increase in PI4P5K activity was a non-specific effect of growth at elevated temperatures, cells were induced to form germ tubes by the combination of elevated temperature and serum. The

Table 1. Percentage amino acid similarity of full-length members of the PI4P5K family

Abbreviations and accession numbers are as follows: Arabidopsis thaliana (At), T51821; C. albicans (Ca), CaMSS4; Caenorhabditis elegans (Ce), CAA91791.1; Drosophila melanogaster (Dm), AAC25576.1; Homo sapiens alpha (Hs $\alpha$ ), XP_001338.2; Homo sapiens beta (Hs $\beta)$, XP_015261.1; Mus musculus alpha (Mm $\alpha$ ), NP_032872.1; Mus musculus beta (Mm $\beta)$, NP_032873.1; Mus musculus gamma (Mm $)$, NP_032870.1; Nicotiana rustica (Nr), AAF80332.1; Oryza sativa (Os), AAG60194.1; Saccharomyces cerevisiae (Sc), P38994; Schizosaccharomyces pombe (Sp), CAB10125.1; Sus scrofa domestica (Ssd), AAB53645.1.

\begin{tabular}{|c|c|c|c|c|c|c|c|c|c|c|c|c|c|c|}
\hline & At & $\mathrm{Ca}$ & $\mathrm{Ce}$ & $\mathrm{Dm}$ & $\mathrm{Hs} \alpha$ & $\mathrm{Hs} \boldsymbol{\beta}$ & $\operatorname{Mm} \alpha$ & $\operatorname{Mm} \beta$ & $\operatorname{Mm} \gamma$ & $\mathrm{Nr}$ & Os & $\mathrm{Sc}$ & $S p$ & Ssd \\
\hline At & - & $25 \cdot 8$ & $11 \cdot 1$ & $18 \cdot 9$ & $22 \cdot 2$ & $19 \cdot 2$ & $21 \cdot 5$ & $20 \cdot 9$ & $17 \cdot 2$ & $74 \cdot 8$ & $14 \cdot 3$ & $17 \cdot 3$ & $20 \cdot 9$ & $20 \cdot 4$ \\
\hline $\mathrm{Ca}$ & & - & $12 \cdot 3$ & $23 \cdot 9$ & $22 \cdot 3$ & $21 \cdot 5$ & $21 \cdot 9$ & $22 \cdot 1$ & $21 \cdot 1$ & $27 \cdot 8$ & $10 \cdot 1$ & $48 \cdot 5$ & $45 \cdot 2$ & $24 \cdot 1$ \\
\hline $\mathrm{Ce}$ & & & - & $11 \cdot 3$ & $10 \cdot 9$ & $11 \cdot 0$ & $11 \cdot 7$ & $11 \cdot 2$ & $12 \cdot 1$ & $10 \cdot 4$ & $10 \cdot 5$ & $10 \cdot 7$ & $11 \cdot 7$ & $12 \cdot 3$ \\
\hline $\mathrm{Dm}$ & & & & - & $49 \cdot 2$ & $41 \cdot 5$ & $44 \cdot 2$ & $46 \cdot 3$ & $38 \cdot 7$ & $19 \cdot 6$ & $10 \cdot 5$ & $15 \cdot 1$ & $18 \cdot 7$ & $19 \cdot 7$ \\
\hline $\mathrm{Hs} \alpha$ & & & & & - & $59 \cdot 9$ & $60 \cdot 1$ & $91 \cdot 4$ & $65 \cdot 8$ & $23 \cdot 5$ & $10 \cdot 5$ & $22 \cdot 0$ & $24 \cdot 3$ & $24 \cdot 1$ \\
\hline $\mathrm{Hs} \beta$ & & & & & & - & $95 \cdot 9$ & $56 \cdot 2$ & $57 \cdot 6$ & $18 \cdot 8$ & $10 \cdot 3$ & $17 \cdot 3$ & $18 \cdot 3$ & $23 \cdot 4$ \\
\hline $\operatorname{Mm} \alpha$ & & & & & & & - & $56 \cdot 6$ & $60 \cdot 3$ & $19 \cdot 9$ & $9 \cdot 4$ & $18 \cdot 6$ & $22 \cdot 1$ & $23 \cdot 2$ \\
\hline $\operatorname{Mm} \beta$ & & & & & & & & - & $63 \cdot 4$ & $22 \cdot 0$ & $10 \cdot 3$ & $19 \cdot 8$ & $23 \cdot 6$ & $23 \cdot 4$ \\
\hline $\operatorname{Mm} \gamma$ & & & & & & & & & - & $17 \cdot 7$ & $12 \cdot 4$ & $14 \cdot 5$ & $19 \cdot 2$ & $23 \cdot 6$ \\
\hline $\mathrm{Nr}$ & & & & & & & & & & - & $17 \cdot 3$ & $18 \cdot 5$ & $20 \cdot 5$ & $22 \cdot 4$ \\
\hline Os & & & & & & & & & & & - & $11 \cdot 1$ & $11 \cdot 1$ & $11 \cdot 3$ \\
\hline $\mathrm{Sc}$ & & & & & & & & & & & & - & $31 \cdot 0$ & $24 \cdot 6$ \\
\hline$S p$ & & & & & & & & & & & & & - & $22 \cdot 9$ \\
\hline Ssd & & & & & & & & & & & & & & - \\
\hline
\end{tabular}



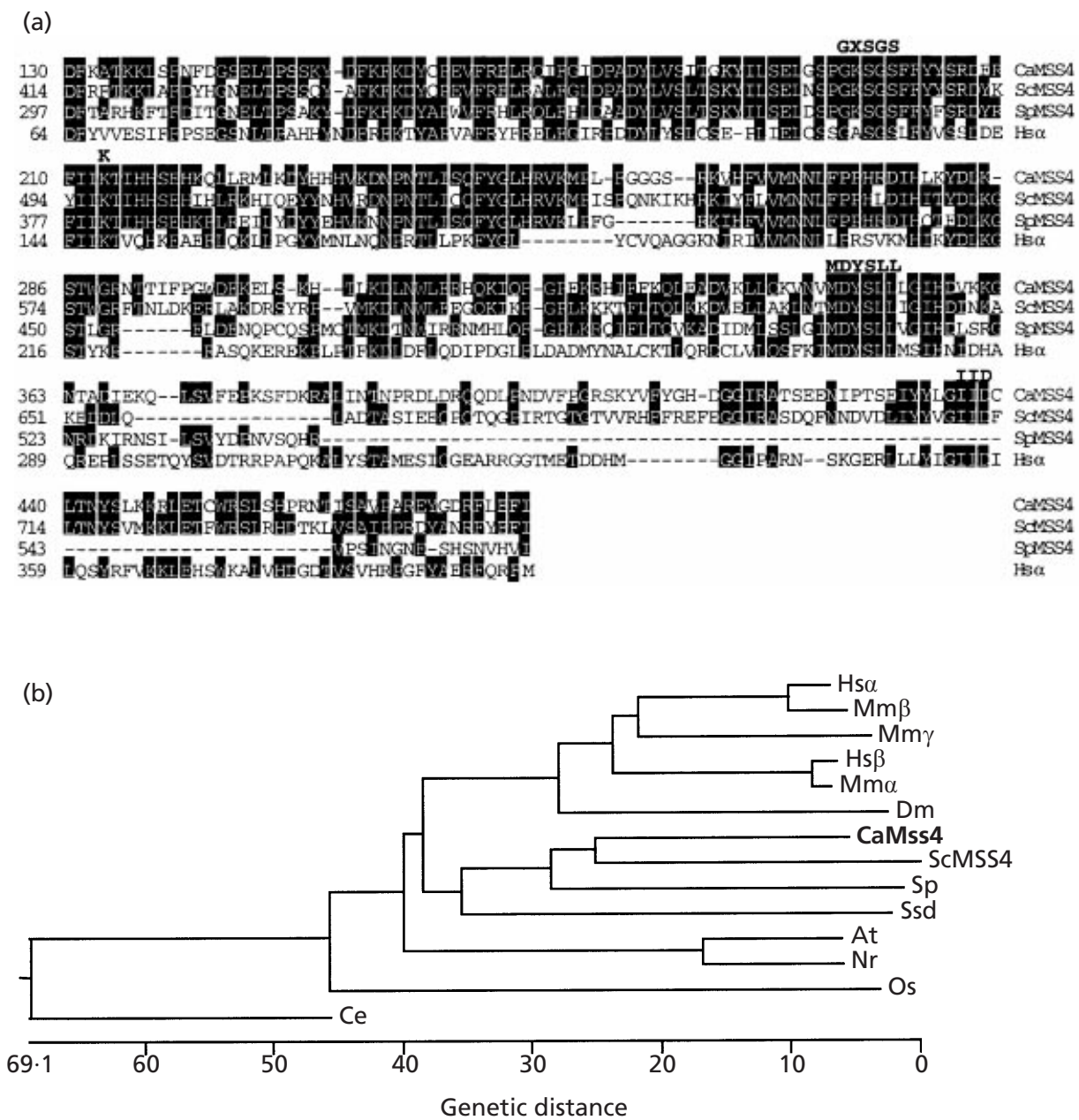

Fig. 5. C. albicans PI4P5K protein is homologous to PI4P5K family members. (a) Alignment of the kinase homology domains of selected PI4P5K family members. Highly conserved residues are shown on a black background. Amino acid residue numbers are annotated on the left. GXSGS, a putative nucleotide-binding motif; MDYSL, a moiety important for catalysis; and IID, a putative $\mathrm{Mg}^{2+}$ - and $\mathrm{Mn}^{2+}$-binding motif are indicated above each consensus sequence. (b) Phylogenetic tree for PI4P5Ks of various species. Horizontal distances correspond to genetic distance. See Table 1 for the percentage amino acid similarity of full-length members of the PI4P5K family.

results of a representative experiment are shown in Fig. 2 (b). As expected, the kinetics and extent of germ-tube formation was nearly identical to that seen with temperature alone (Fig. 2a). PI4P5K activity responded to the complex stimuli in a qualitatively similar manner: activity increased during the first $20 \mathrm{~min}$, declined and rose again at 90 min post-induction. However, unlike the results with temperature stimulation alone, the quantitative changes induced by temperature and serum lacked statistical significance.

\section{Propranolol inhibits PI4P5K induction during morphogenesis}

To determine whether PA was activating PI4P5K in vivo, propranolol was added to cultures coincident with the induction of morphogenesis. Propranolol has been used at low concentrations $(0 \cdot 1-0 \cdot 2 \mathrm{mM})$ to inhibit mammalian lipid phosphate phosphohydrolases (LPPs) that convert PA into DAG. The LPPs in C. albicans are not affected by low concentrations of propranolol (C. A. Baker, K. Desrosiers \& J. W. Dolan, unpublished); consequently, higher concentrations (1-5 mM) were used. At the higher concentrations, propranolol can act by binding to the PA and rendering it inaccessible to downstream targets of PA (C. A. Baker, K. Desrosiers \& J. W. Dolan, unpublished; Surewicz \& Leyko, 1981). As expected, propranolol attenuated PI4P5K stimulation throughout the dimorphic transition (Fig. 3a) relative to untreated controls (Fig. 2a). The addition of $2 \mathrm{mM}$ propranolol also eliminated germ-tube formation in these cultures (Fig. 3a). To rule out the possibility that the inhibition of germ-tube formation was the result of reduced proliferation, growth rates were determined at increasing propranolol concentrations. The addition of propranolol in concentrations up to $1 \mathrm{mM}$ did not affect growth rates. However, $2 \mathrm{mM}$ caused a slight increase in generation time (Fig. 3b). Thus, propranolol reduced PI4P5K activation and germ-tube formation without substantially affecting the growth rate. 


\section{Correlation of PI4P5K and PLD1 activity}

In light of the fact that both PLD1 and PI4P5K are activated during morphogenesis (McLain \& Dolan, 1997), PLD1 activity was assayed in the same extracts used to determine PI4P5K activation (Fig. 2a). The timing of activation revealed that PI4P5K activity is stimulated earlier than PLD1 and to a larger degree, suggesting that $\mathrm{PIP}_{2}$ generated by PI4P5K could account for the subsequent stimulation of PLD1.

To determine whether PLD1 was playing a role in the stimulation of PI4P5K, a homozygous pld1s knockout strain, JDC12 (Hube et al., 2001), was assayed for PI4P5K activity during morphogenesis. PI4P5K activity was observed to decline steadily during morphogenesis in the pld $1 \Delta$ cells (Fig. 4). Although PA can be generated by the phosphorylation of DAG by diglyceride kinase (Morlock et al., 1991; Wu et al., 1993) and by acylation of monoacyl-glycerol 3-phosphate (Athenstaedt et al., 1999), this complete lack of induction of PI4P5K activity in pld $1 \Delta$ cells indicates that PLD1 supplies PA necessary for PI4P5K stimulation during dimorphic transition to elongated hyphae.

\section{C. albicans carries a gene that is highly homologous to PI4P5K family members}

An ORF on contig 6-1966 was found by a BLAST search of the C. albicans sequencing project (http://wwwsequence.standford.edu/group/candida) that was predicted to encode a protein closely related to Mss4 $\mathrm{p}$ of $S$. cerevisiae. The ORF is 3326 bp in length, encodes 491 aa and is $54 \%$ identical and $69 \%$ similar to the $S$. cerevisiae protein over a region of 508 aa of the $S$. cerevisiae protein (Table 1). A region of 347 aa contains several motifs typical of kinases. Of the 347 aligned residues, 31 are conserved in all PI4P5K homologues examined; an alignment of this region from three fungal homologues and one human homologue is shown in Fig. 5(a). These conserved residues are likely to be involved in phosphotransferase activity or are strategically located to interact with the substrate, PI4P, in the active site. C. albicans PI4P5K has a glycine-rich loop with the consensus sequence of GXSGS (residues 195-199) and a conserved lysine (residue 212). These residues resemble the phosphate-binding loop of protein kinases and other nucleotide-binding proteins (Anderson et al., 1999). As with other kinases, C. albicans and other PI4P5K family members share an aspartic acid (D) residue (347 in $C$. albicans) within the consensus sequence MDYSL. This aspartic acid residue corresponds to D166, which is important for catalysis in protein kinase A (Knighton et al., 1991). Another aspartic acid residue (436) is present within the consensus sequence IID in the C. albicans protein and other homologues and coincides with D184 in protein kinase $\mathrm{A}$ that binds $\mathrm{Mg}^{2+}$ and $\mathrm{Mn}^{2+}$ ions (Knighton et al., 1991).

A phylogenetic tree was generated based on the homology of kinase domains of known or suspected PI4P5Ks to better understand the relationships within this gene (a)

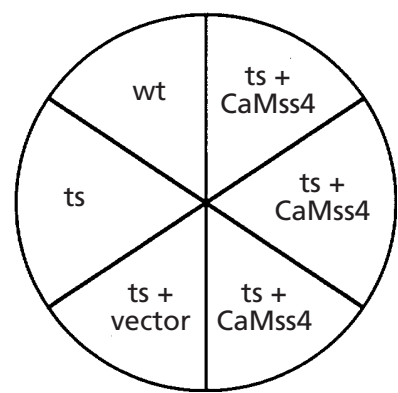

(b)
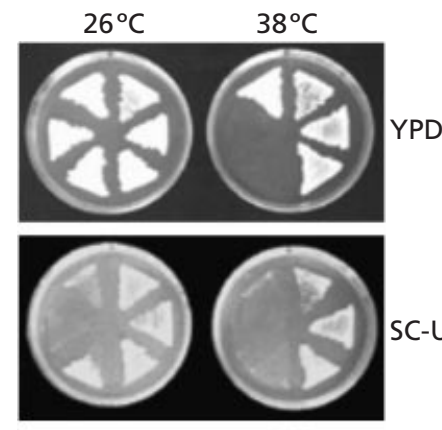

SC-Ura
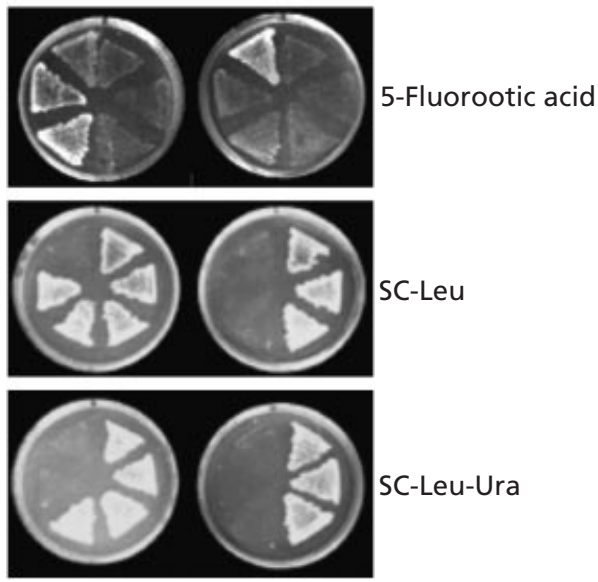

Fig. 6. The $C$. albicans homologue of MSS4 suppresses the temperature-sensitivity of an $S$. cerevisiae mss $^{\text {ts }}$ mutant. Relevant phenotypes of tested strains are shown in Table 2. (a) Key to strains tested for growth at permissive $\left(26^{\circ} \mathrm{C}\right)$ and non-permissive $\left(38^{\circ} \mathrm{C}\right)$ temperatures. The 'ts + CaMSS4' strains represent three isolates carrying the plasmid pCW20. (b) Strains were tested for growth on the indicated media. Photographs were taken after 2 days of incubation.

family (Fig. 5b). The tree contained three prominent clusters. The first cluster was composed of fungal homologues, including C. albicans Mss4p, as well as the porcine kinase. The second cluster contained human, mouse and Drosophila melanogaster PI4P5Ks. The third cluster contained plant homologues.

\section{C. albicans MSS4 can complement a S. cerevisiae mss $4^{\text {ts }}$}

To determine whether the cloned ORF encodes a functional homologue of the S. cerevisiae PI4P5K Mss4p, we constructed a plasmid ( $\mathrm{pCW} 20$ ) carrying this gene 
Table 2. Relevant phenotypes of tested strains

\begin{tabular}{|lccc|}
\hline Strain & Leu & Ura & Mss4 \\
\hline Wild-type (wt) & - & - & + \\
mss44 YEplac-mss4-102 & + & - & ts \\
mss44 YEplac-mss4-102 YEp352 & + & + & ts \\
$m s s 4 \Delta$ YEplac-mss4-102 pCW20 & + & + & CaMSS4 \\
\hline
\end{tabular}

under the transcriptional control of its own putative promoter. The amino acid sequence has a serine residue in the $\mathrm{C}$ terminus at position 463 that is encoded by nonstandard CUG codon usage in C. albicans (Ohama et al., 1993; Santos et al., 1993). pCW20 was transformed into S. cerevisiae strains SEY6210.1 (MSS4) and AAY202.1 ( $m s s 4 \Delta:$ :HIS3 carrying YEplac-mss $4-102^{\text {ts }}$ ) and the transformants were tested for the ability to suppress the temperature sensitivity of the mss $4-102$ allele (Fig. 6 and Table 2). The temperature-sensitive transformants carrying pCW20, which contains the URA3 gene as a selectable marker, were unable to grow on plates containing 5-fluoroorotic acid, indicating that the Candida MSS4 gene was required for viability at the non-permissive temperature $\left(38^{\circ} \mathrm{C}\right)$. Since these strains carried the mss4-102 temperature-sensitive allele, the cells should be able to lose pCW20 and grow on 5fluoroorotic acid at the permissive temperature of $26^{\circ} \mathrm{C}$. The pCW20 transformants were able to grow on 5fluoroorotic acid at $26^{\circ} \mathrm{C}$, forming colonies after extended incubation (data not shown). The slow growth suggests that even at the permissive temperature, the mss $4-102$ cells benefit from the added activity provided by the C. albicans MSS4 gene on pCW20. Plasmid pCW20 was also used to transform $S$. cerevisiae strain 23567, a diploid that is heterozygous for MSS4 (MSS4/ $\left.m s s 4:: \mathrm{Kan}^{\mathrm{R}}\right)$. These transformants showed an approximately $40 \%$ increase in PI4P5K activity that returned to untransformed levels following loss of pCW20. Taken together, these results confirm that the C. albicans gene designated MSS4 encodes a functional PI4P5K. The presence of additional C. albicans genes encoding PI4P5K activity cannot be excluded, but no other regions with significant homology to PI4P5Ks have been identified in the genome sequence.

\section{DISCUSSION}

In the present study, PI4P5K activity in the pathogenic fungus, C. albicans was identified. As in S. cerevisiae (Desrivieres et al., 1998; Homma et al., 1998), murine and bovine cells (Ishihara et al., 1996, 1998; Jenkins et al., 1994; Moritz et al., 1992), C. albicans PI4P5K is stimulated by PA. In addition, we have found this PI4P5K, like other known PI4P5Ks (Anderson et al., 1999; Homma et al., 1998; Honda et al., 1999; Vancurova et al., 1999), is membrane-associated (Fig. 1). A gene, designated MSS4, has been cloned and demonstrated to encode a PI4P5K activity when expressed in S. cerevisiae.
Our results suggest that PI4P5K might play a role in C. albicans transition to polarized growth in response to some, but not all, inducing stimuli. The exact role PI4P5K would play in this process is unknown; however, the change of shape from an ellipsoid to an elongated germ tube involves actin cytoskeleton reorganization. The product of PI4P5K activity, $\mathrm{PIP}_{2}$, could affect cell polarization in a number of ways. For example, $\mathrm{PIP}_{2}$ has been identified as a lipid that dissociates capping proteins from the barbed ends of actin filaments, thus promoting actin assembly (Heiss \& Cooper, 1991; Janmey et al., 1987; Janmey \& Stossel, 1987). Profilin is an actin-binding protein that binds PIP $_{2}$. It is required for the maintenance of cell polarity and is essential for the proper formation of actin cables that occur at areas of active cellular growth (DiNubile \& Huang, 1997a, b). Thus, PI4P5Ks in yeast and murine models have been shown to be critical for actin assembly (Audhya et al., 2000; Desrivieres et al., 1998; Homma et al., 1998; Tolias et al., 2000). It is therefore plausible that $C$. albicans PI4P5K is stimulated to provide increased levels of $\mathrm{PIP}_{2}$ for the actin remodelling process.

PI4P5K stimulation during morphogenesis is attenuated by the addition of propranolol (Fig. 3). Propranolol is typically used in mammalian cells at low concentrations $(100-200 \mu \mathrm{M})$ to inhibit LPP, the enzyme that hydrolyses PA to DAG (Meier et al., 1998; Morlock et al., 1991; Wu et al., 1993). Frequently, inhibition by propranolol suggests that DAG, rather than PA, is the essential molecule in a cellular process. In contrast to mammalian LPP, yeast LPP is relatively insensitive to propranolol (C. A. Baker, K. Desrosiers \& J. W. Dolan, unpublished; Ella et al., 1995). An earlier study using fluorescent spectroscopy and millimolar concentrations of propranolol revealed that the drug bound directly to several phospholipids, particularly PA and phosphatidylserine (Surewicz \& Leyko, 1981). In addition, fluorimetry studies have shown that the intrinsic fluorescence of propranolol is quenched by PA in a concentrationdependent mode (C. A. Baker, K. Desrosiers \& J. W. Dolan, unpublished). The change in fluorescence at high PA concentrations suggests that propranolol is acting by binding to and sequestering PA, making the PA unavailable for subsequent activity by LPP, PI4P5K or any other downstream target. Furthermore, when DAG levels were measured in wild-type C. albicans cells in response to millimolar concentrations of the drug, total cellular DAG levels remained unchanged compared to untreated cells (C. A. Baker, K. Desrosiers \& J. W. Dolan, unpublished). These results, taken together, indicate that PA, rather than DAG, is the important molecule derived from PLD1 activity during morphogenesis.

In this study, the data suggest PLD1 is the source of PA required to stimulate PI4P5K during morphogenesis. Our results provide further evidence for a role of PLD1 in polarized growth (Hube et al., 2001; McLain \& Dolan, 1997) and a possible role for PI4P5K in this process. As shown in Fig. 2(a), PI4P5K is highly activated when germ tubes begin to emerge from pre-existing 
cells, whereas PLD1 activity does not peak until later. This timing would suggest that $\mathrm{PIP}_{2}$ formed by the action of PI4P5K stimulates PLD1. There is, however, a highly reproducible $10 \%$ increase in PLD1 activity 10 min after induction of morphogenesis before any germ tubes have emerged (Fig. 2a; McLain \& Dolan, 1997). It is possible that PA is generated by stimulation of a highly localized pool of PLD1 to initially activate PI4P5K. Supporting this idea, PI4P5K activity was attenuated in extracts prepared from pld $1 \Delta$ null mutant cells and by the addition of propranolol. Interestingly, germ-tube formation in pld1 $\Delta$ cells was unaffected by the lack of PLD1 signalling and PI4P5K stimulation. Similarly, germ tubes were formed normally in response to induction by both serum and temperature, even though PI4P5K activity was relatively unaffected. These results were not unexpected, due to the multiple pathways that can induce hyphal development (Ernst, 2000). Thus, $\mathrm{PIP}_{2}$ may be important to morphogenesis in response to some stimuli and irrelevant in response to other stimuli. Further studies are needed to determine whether the increase in PI4P5K activity is a cause or consequence of temperature-induced morphogenesis.

\section{ACKNOWLEDGEMENTS}

We would like to thank Dr Bernhard Hube (Robert Koch Institute, Berlin, Germany) for the C. albicans strains used in this study and Dr Scott Emr (Univ. of California, San Diego, CA, USA) for S. cerevisiae strains. We would also like to thank Dr Michael Yaffe (Univ. of California, San Diego, CA, USA) for providing laboratory space and advice. This work was supported by MUSC Institutional Funds for Research and the American Cancer Society (IRG-97-151-0-IRG).

\section{REFERENCES}

Amsterdam, A., Dantes, A. \& Liscovitch, M. (1994). Role of phospholipase-D and phosphatidic acid in mediating gonadotropin-releasing hormone-induced inhibition of preantral granulosa cell differentiation. Endocrinology 135, 1205-1211.

Anderson, R. A., Boronenkov, I. V., Doughman, S. D., Kunz, J. \& Loijens, J. C. (1999). Phosphatidylinositol phosphate kinases, a multifaceted family of signaling enzymes. J Biol Chem 274, 9907-9910.

Athenstaedt, K., Weys, S., Paltauf, F. \& Daum, G. (1999). Redundant systems of phosphatidic acid biosynthesis via acylation of glycerol-3-phosphate or dihydroxyacetone phosphate in the yeast Saccharomyces cerevisiae. J Bacteriol 181, 1458-1463.

Audhya, A., Foti, M. \& Emr, S. D. (2000). Distinct roles for the yeast phosphatidylinositol 4-kinases, Stt4p and Pik1p, in secretion, cell growth, and organelle membrane dynamics. Mol Biol Cell 11, 2673-2689.

Bligh, E. C. \& Dyer, W. J. (1959). A rapid method of total lipid extraction and purification. Can J Biochem Physiol 37, 911-917.

Chong, L. D., Traynor-Kaplan, A., Bokoch, G. M. \& Schwartz, M. A. (1994). The small GTP-binding protein Rho regulates a phosphatidylinositol 4-phosphate 5-kinase in mammalian cells. Cell 79, 507-513.

Cochet, C. \& Chambaz, E. M. (1986). Catalytic properties of a purified phosphatidylinositol-4-phosphate kinase from rat brain. Biochem J 237, 25-31.
Cutler, J. E. (1991). Putative virulence factors of Candida albicans. Annu Rev Microbiol 45, 187-218.

Desrivieres, S., Cooke, F. T., Parker, P. J. \& Hall, M. N. (1998). MSS4, a phosphatidylinositol-4-phosphate 5-kinase required for organization of the actin cytoskeleton in Saccharomyces cerevisiae. J Biol Chem 273, 15787-15793.

DiNubile, M. J. \& Huang, S. (1997a). Capping of the barbed ends of actin filaments by a high-affinity profilin-actin complex. Cell Motil Cytoskeleton 37, 211-225.

DiNubile, M. J. \& Huang, S. (1997b). High concentrations of phosphatidylinositol-4,5-bisphosphate may promote actin filament growth by three potential mechanisms: inhibiting capping by neutrophil lysates, severing actin filaments and removing capping protein-beta2 from barbed ends. Biochim Biophys Acta 1358, 261-278.

Dove, S. K., Cooke, F. T., Douglas, M. R., Sayers, L. G., Parker, P. J. \& Michell, R. H. (1997). Osmotic stress activates phosphatidylinositol-3,5-bisphosphate synthesis. Nature 390, 187-192.

Ella, K. M., Dolan, J. W. \& Meier, K. E. (1995). Characterization of a regulated form of phospholipase D in the yeast Saccharomyces cerevisiae. Biochem J 307, 799-805.

Ernst, J. F. (2000). Regulation of dimorphism in Candida albicans. Contrib Microbiol 5, 98-111.

Fruman, D. A., Meyers, R. E. \& Cantley, L. C. (1998). Phosphoinositide kinases. Annu Rev Biochem 67, 481-507.

Fukami, K., Furuhashi, K., Inagaki, M., Endo, T., Hatano, S. \& Takenawa, T. (1992). Requirement of phosphatidylinositol 4,5bisphosphate for alpha-actinin function. Nature 359, 150-152.

Gietz, R. D. \& Woods, R. A. (2001). Genetic transformation of yeast. Biotechniques 30, 816-820, 822-826, 828 passim.

Ha, K. S. \& Exton, J. H. (1993). Activation of actin polymerization by phosphatidic acid derived from phosphatidylcholine in IIC9 fibroblasts. J Cell Biol 123, 1789-1796.

Heiss, S. G. \& Cooper, J. A. (1991). Regulation of CapZ, an actin capping protein of chicken muscle, by anionic phospholipids. Biochemistry 30, 8753-8758.

Homma, K., Terui, S., Minemura, M., Qadota, H., Anraku, Y., Kanaho, Y. \& Ohya, Y. (1998). Phosphatidylinositol-4-phosphate 5 -kinase localized on the plasma membrane is essential for yeast cell morphogenesis. J Biol Chem 273, 15779-15786.

Honda, A., Nogami, M., Yokozeki, T. \& 8 other authors (1999). Phosphatidylinositol 4-phosphate 5-kinase alpha is a downstream effector of the small G protein ARF6 in membrane ruffle formation. Cell 99, 521-532.

Hube, B., Hess, D., Baker, C. A., Schaller, M., Schafer, W. \& Dolan, J. W. (2001). The role and relevance of phospholipase D1 during growth and dimorphism of Candida albicans. Microbiology 147, 879-889.

Isakoff, S. J., Cardozo, T., Andreev, J., Li, Z., Ferguson, K. M., Abagyan, R., Lemmon, M. A., Aronheim, A. \& Skolnik, E. Y. (1998). Identification and analysis of $\mathrm{PH}$ domain-containing targets of phosphatidylinositol 3-kinase using a novel in vivo assay in yeast. EMBO J 17, 5374-5387.

Ishihara, H., Shibasaki, Y., Kizuki, N., Katagiri, H., Yazaki, Y., Asano, T. \& Oka, Y. (1996). Cloning of cDNAs encoding two isoforms of $68-\mathrm{kDa}$ type I phosphatidylinositol-4-phosphate 5kinase. J Biol Chem 271, 23611-23614.

Ishihara, H., Shibasaki, Y., Kizuki, N., Wada, T., Yazaki, Y., Asano, T. \& Oka, Y. (1998). Type I phosphatidylinositol-4-phosphate 5kinases. Cloning of the third isoform and deletion/substitution analysis of members of this novel lipid kinase family. J Biol Chem 273, 8741-8748. 
Janmey, P. A. (1994). Phosphoinositides and calcium as regulators of cellular actin assembly and disassembly. Annu Rev Physiol 56, 169-191.

Janmey, P. A. \& Stossel, T. P. (1987). Modulation of gelsolin function by phosphatidylinositol 4, 5-bisphosphate. Nature 325, 362-364.

Janmey, P. A., lida, K., Yin, H. L. \& Stossel, T. P. (1987). Polyphosphoinositide micelles and polyphosphoinositide-containing vesicles dissociate endogenous gelsolin-actin complexes and promote actin assembly from the fast-growing end of actin filaments blocked by gelsolin. J Biol Chem 262, 12228-12236.

Jenkins, G. H., Fisette, P. L. \& Anderson, R. A. (1994). Type I phosphatidylinositol 4-phosphate 5-kinase isoforms are specifically stimulated by phosphatidic acid. J Biol Chem 269, 11547-11554.

Kaszkin, M., Richards, J. \& Kinzel, V. (1996). Phosphatidic acid mobilized by phospholipase D is involved in the phorbol 12myristate 13-acetate-induced G2 delay of A431 cells. Biochem J 314, 129-138.

Knighton, D. R., Zheng, J. H., Ten Eyck, L. F., Ashford, V. A., Xuong, N. H., Taylor, S. S. \& Sowadski, J. M. (1991). Crystal structure of the catalytic subunit of cyclic adenosine monophosphate-dependent protein kinase. Science 253, 407-414.

Leberer, E., Ziegelbauer, K., Schmidt, A., Harcus, D., Dignard, D., Ash, J., Johnson, L. \& Thomas, D. Y. (1997). Virulence and hyphal formation of Candida albicans require the Ste20p-like protein kinase CaCla4p. Curr Biol 7, 539-546.

Liscovitch, M., Chalifa, V., Pertile, P., Chen, C. S. \& Cantley, L. C. (1994). Novel function of phosphatidylinositol 4,5-bisphosphate as a cofactor for brain membrane phospholipase D. J Biol Chem 269, 21403-21406.

Liscovitch, M., Czarny, M., Fiucci, G., Lavie, Y. \& Tang, X. (1999). Localization and possible functions of phospholipase D isozymes. Biochim Biophys Acta 1439, 245-263.

Loijens, J. C. \& Anderson, R. A. (1996). Type I phosphatidylinositol-4-phosphate 5-kinases are distinct members of this novel lipid kinase family. J Biol Chem 271, 32937-32943.

Loijens, J. C., Boronenkov, I. V., Parker, G. J. \& Anderson, R. A. (1996). The phosphatidylinositol 4-phosphate 5-kinase family. Adv Enzyme Regul 36, 115-140.

Majerus, P. W. (1992). Inositol phosphate biochemistry. Annu Rev Biochem 61, 225-250.

McLain, N. \& Dolan, J. W. (1997). Phospholipase D activity is required for dimorphic transition in Candida albicans. Microbiology 143, 3521-3526.

Meier, K. E., Gause, K. C., Wisehart-Johnson, A. E., Gore, A. C., Finley, E. L., Jones, L. G., Bradshaw, C. D., McNair, A. F. \& Ella, K. M. (1998). Effects of propranolol on phosphatidate phosphohydrolase and mitogen-activated protein kinase activities in A7r5 vascular smooth muscle cells. Cell Signal 10, 415-426.

Moritz, A., De Graan, P. N., Gispen, W. H. \& Wirtz, K. W. (1992). Phosphatidic acid is a specific activator of phosphatidylinositol-4phosphate kinase. J Biol Chem 267, 7207-7210.

Morlock, K. R., McLaughlin, J. J., Lin, Y. P. \& Carman, G. M. (1991). Phosphatidate phosphatase from Saccharomyces cerevisiae. Isolation of 45 - and $104-\mathrm{kDa}$ forms of the enzyme that are differentially regulated by inositol. J Biol Chem 266, 3586-3593.
Navarro-Garcia, F., Sanchez, M., Nombela, C. \& Pla, J. (2001). Virulence genes in the pathogenic yeast Candida albicans. FEMS Microbiol Rev 25, 245-268.

Odds, F. C. (1988). Candida and Candidosis, 2nd edn. Philadelphia, PA: Bailliere Tindall.

Odorizzi, G., Babst, M. \& Emr, S. D. (2000). Phosphoinositide signaling and the regulation of membrane trafficking in yeast. Trends Biochem Sci 25, 229-235.

Ohama, T., Suzuki, T., Mori, M., Osawa, S., Ueda, T., Watanabe, K. \& Nakase, T. (1993). Non-universal decoding of the leucine codon CUG in several Candida species. Nucleic Acids Res 21, 4039-4045.

Rameh, L. E., Tolias, K. F., Duckworth, B. C. \& Cantley, L. C. (1997). A new pathway for synthesis of phosphatidylinositol-4,5-bisphosphate. Nature 390, 192-196.

Rex, J. H., Rinaldi, M. G. \& Pfaller, M. A. (1995). Resistance of Candida species to fluconazole. Antimicrob Agents Chemother 39, 1-8.

Rose, K., Rudge, S. A., Frohman, M. A., Morris, A. J. \& Engebrecht, J. (1995). Phospholipase D signaling is essential for meiosis. Proc Natl Acad Sci US A 92, 12151-12155.

Santos, M. A., Keith, G. \& Tuite, M. F. (1993). Non-standard translational events in Candida albicans mediated by an unusual seryl-tRNA with a 5'-CAG-3(leucine) anticodon. EMBO J 12, 607-616.

Sherman, F. (1991). Getting started with yeast. Methods Enzymol 194, 3-21.

Siddhanta, A. \& Shields, D. (1998). Secretory vesicle budding from the trans-Golgi network is mediated by phosphatidic acid levels. J Biol Chem 273, 17995-17998.

Surewicz, W. K. \& Leyko, W. (1981). Interaction of propranolol with model phospholipid membranes. Monolayer, spin label and fluorescent spectroscopy studies. Biochim Biophys Acta 643, 387-397.

Tolias, K. F., Hartwig, J. H., Ishihara, H., Shibasaki, Y., Cantley, L. C. \& Carpenter, C. L. (2000). Type I alpha phosphatidylinositol4-phosphate 5-kinase mediates Rac-dependent actin assembly. Curr Biol 10, 153-156.

Vancurova, I., Choi, J. H., Lin, H., Kuret, J. \& Vancura, A. (1999). Regulation of phosphatidylinositol 4-phosphate 5-kinase from Schizosaccharomyces pombe by casein kinase I. J Biol Chem 274, 1147-1155.

Varnai, P. \& Balla, T. (1998). Visualization of phosphoinositides that bind pleckstrin homology domains: calcium-and agonistinduced dynamic changes and relationship to myo- $\left[{ }^{3} \mathrm{H}\right]$ inositollabeled phosphoinositide pools. J Cell Biol 143, 501-510.

Waksman, M., Eli, Y., Liscovitch, M. \& Gerst, J. E. (1996). Identification and characterization of a gene encoding phospholipase D activity in yeast. J Biol Chem 271, 2361-2364.

Wu, W. I., Lin, Y. P., Wang, E., Merrill, A. H., Jr \& Carman, G. M. (1993). Regulation of phosphatidate phosphatase activity from the yeast Saccharomyces cerevisiae by sphingoid bases. J Biol Chem 268, 13830-13837.

Received 7 August 2001; revised 11 February 2002; accepted 25 February 2002. 